\title{
COMPARISON OF TRUNK AND LEG SWAY DURING SINGLE LEG STANCE
}

\author{
Howe Liu ${ }^{* 1}$, Yasser Salem ${ }^{2}$, Jingjie Zhou ${ }^{3}$, Clayton Holmes ${ }^{4}$, Myla Quiben ${ }^{5}$. \\ ${ }^{*} 1,2,5$ Department of Physical Therapy, University of North Texas Health Science Center, Fort Worth, \\ Texas, USA. \\ ${ }^{3}$ Department of Rehabilition Service, Xuzhou Rehabilitation Hospital, Xuzhou, China. \\ ${ }^{4}$ School of Physical Therapy, Arkansas College of Healthcare Education, Fort Smith, AR. USA.
}

\section{ABSTRACT}

Background: Singe leg stance (SLS) is a commonly used assessment of balance, but there is lack of knowledge of how different body part may be involved in the SLS maintenance. The purpose of this study was to utilize small inertial measurement unit (IMUs) to investigate how different body segments respond during static SLS.

Methods: This was a cross-sectional study with two IMU sensors utilized to compare two body locations. The sensors were placed at two flat areas - on L4-5 spinous processes for the trunk segment, and at the left popliteal fossa immediately below the knee joint line for the leg segment. All subjects recruited had the left leg as the nondominant leg. These subjects were asked to perform a SLS on a flat hard surface with their non-dominant leg. Subjects held this position for 30 seconds, while data of body sway parameters (range, angular velocity, and acceleration) were recorded and transmitted wirelessly to a computer for storage and analysis.

Results: Compared with the leg sway, the trunk displaying a larger range in faster speed and greater acceleration than the leg primarily in the sagittal (anterior-posterior) direction (all $p<.05$ ). Also, quicker speed in axial plane, and greater acceleration in both axial and frontal planes were observed in trunk than in leg ( $p<.05)$; but no obvious differences were identified in range of sway in these two planes ( $P>05)$.

Conclusions. During SLS on the non-dominant left leg, the whole body stays more toward left in frontal and axial planes but sways more in sagittal plane. These data may provide baseline information for future studies in more body segments and in elderly people as well.

KEY WORDS: body segments, balance, inertial sensor, leg, trunk, 3-D planes.

Address for correspondence: Howe Liu, MPT, PhD, MS, MD, Department of Physical Therapy, University of North Texas Health Science Center, 3500 Camp Bowie Blvd. Fort Worth, Texas 76107, USA. E-Mail: Howe.Liu@unthsc.edu

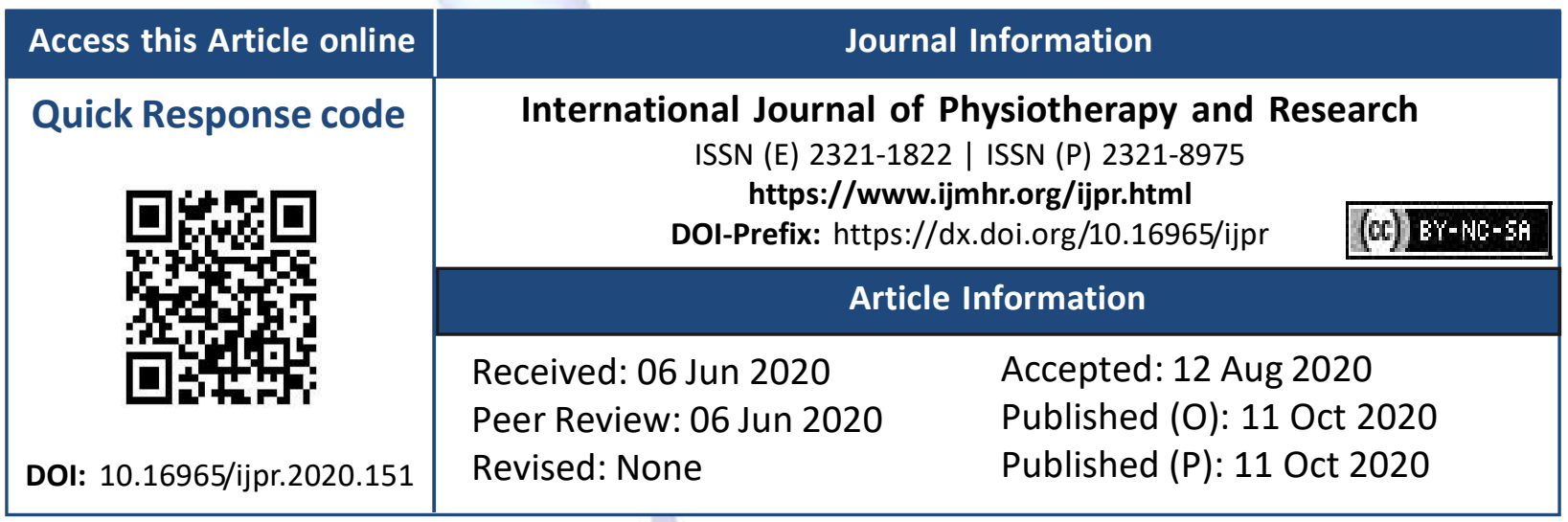

\section{INTRODUCTION}

As an assessment for both static and dynamic balance, single leg stance (SLS) is commonly used by clinicians in clinical and community settings to evaluate a person's motor function and balance $[1,2]$. Ability to stand on a single leg with confidence is critical for one to hold a proper posture against gravity in order to perform many activities of daily living (ADL), for example, when one puts an item to an overhead 
shelf. During a gait cycle, the stability of one leg during stance phase (namely the SLS period) is also the pre-condition of swing phase and foot initiation of the opposite leg, which can be achieved by keeping the whole body's center of gravity within the base of support formed by the bottom of the standing foot. If one cannot control him/herself while maintaining balance on one leg, it will be very difficult or even incapable of conducting dynamic body movements against gravity. Thus, it is understandable that the static SLS can be the prerequisite of dynamic functionality.

There are internal and external factors that may affect the static balance [3]. Compared with the external factors like hard/soft surface, bright or dim environment which are more controllable, the internal factors including the person's medical, musculoskeletal, proprioceptive, visual, and vestibular conditions may be more challenging to deal with for rehabilitation. Muscle strength, joint flexibility, cardiopulmonary endurance, even the foot positions are all more or less related to sensorimotor musculoskeletal adjustment during balance time.

One of musculoskeletal involvements for balance could be how different body segments participate in the adjustment in response to the body balance. It has been revealed that with multisensory feedbacks to the central nervous system (CNS) and sensorimotor control of CNS, leg and trunk segments could coordinate to maintain balance in different directions [4], angular excursions [5], and postures and activities [5,6]. During the time, body segments might response differently. Wu G, et al (1998)[7] measured the range of motion (ROM) on sagittal plane during the time for involuntary balance in a healthy population (20 to 70 years old), they found that the upper body showed more ROM than the lower body, and the older one aged, the more ROM the upper body demonstrated. Later $\mathrm{Yu} \mathrm{JH}$, et al, (2014) [8] reported that muscles in both upper body (e.g., the trunk) and lower body (e.g., legs) were active in maintaining static standing balance, but the leg muscles played more important role in the task than the trunk muscles.

Standing balance can be defined as one's ability to maintain his/her center of gravity
(COG) within the base of support (BOS) with minimal body sway[9]. The BOS is formed by both feet if one stands bipedally or by the single standing foot if one stands unipedally. Postural sway has been intensively studied regardless of whether subjects stood on both feet $[10,11]$ or on a single foot $[9,12,13]$. However, when postural sway was assessed, force plates were often used in taking the measurements, like ground reaction force, the strategy and velocity of the whole body sway $[14,15]$. In reality, force plates are bulky, costly, and unable to assess the movement of body segments [14-16]. With these being the case, studies have identified the cost-effective, easy-to-manage, wearable, inertial sensors for assessment of postural sway $[12.17,18]$. The wearable inertial measurement units (IMUs) have been demonstrated to be effective and reliable for evaluation of direction, velocity, range, and acceleration of body sway $[17,18,20]$.

IMUs have been extensively used for balance and gait evaluation. A single IMU could be used when an individual's whole body was considered as a single unit; or two or more IMUs could be used when different body segments of the individual were assessed [19-21]. These IMUs were primarily utilized when subjects were during bipedal stance time $[20,21]$, but IMU for subjects in unipedal stance was also reported before [12]. However, so far, application of more than one IMU on persons in single leg standing has not been investigated. Therefore, the purpose of this study was to use a small, wearable IMU-based balance assessment instrument to investigate how different body segments (Lower extremity and trunk) respond during static standing while maintaining a SLS. We believe that results from this project would provide insightful information before we conduct the similar study in older adults.

\section{METHODS}

This was a cross-sectional study design to assess the sway of leg and trunk during single leg stance.

Subjects: This study was conducted in the principle investigator's (PI) research lab. Subjects for the study were recruited through sample of convenience from physical therapy 
graduate students in the Pl's institution. By means of bulletin posts, emails, and classroom announcement with explanations of the study's purpose, inclusion and exclusion criteria (see below), 19 (10 males and 9 females) young healthy participants were qualified and recruited to be subjects. This project was approved by the principle investigator's Institutional Review Board. All subjects were required to sign an informed consent form before being screened for participation in this study based on selection criteria below.

The inclusion criteria included: 1 ). being free of pain or injury within the past six months; 2). be between 20-35 years of age; 3 ). free of any upper and lower extremity, or spinal abnormalities (like contracture, scoliosis, and kyphosis); 4). dominant leg on the right which was defined as the leg being able to comfortably kick a ball when the opposite one was standing [22]; and 5). able to maintain a single leg stance on the left leg for a minimum of 30 seconds on a level surface.

The exclusion criteria were: 1 ). taking any prescribed medication in last 3 months; 2). any ailments that might lead balance deficit (unable to do SLS over 30 seconds); 3). flat foot on the left side measured with the Staheli arch method [23], 4). BMI was over 25 or more, or 5). refusal to sign informed consent.

Instruments: The wearable inertial multisensory unit (IMU) is a single integrated circuit package that consists of a 3-axis gyroscope, 3-axis accelerometer, and 3-axis magnetometer $[17,20,24,25]$. This unit is able to measure postural sway in terms of direction, angular velocity, and linear acceleration in 3-D space $[17,21,25]$. Currently IMUs have been considered as feasible and valid as the force plate for evaluating postural stability, balance, and joint angular movement $[16,20,25]$ with good reliability (Cohen's kappa $=0.79$ ) [20]. In this study, two wearable IMUs (Alda Balance Gear - ABG, Alexandave Industries Co., Taiwan) were utilized for the purpose of the project. Also two laptops were used to receive data wirelessly from each of ABGs.

Procedure: To begin the assessment, investigators established the subject's leg dominance by employing the ball kick method - the leg that kicked the ball as the dominant one [22]. Two ABG devices were secured with straps at two locations on the subject's posterior body: the $1^{\text {st }}$ one at the midline of $4^{\text {th }}-5^{\text {th }}$ lumbar vertebral level to assess trunk sway; and the $2^{\text {nd }}$ one at the popliteal fossa immediately below the knee joint line of the non-dominant leg to assess the lower extremity sway (Figure 1A). Before the formal testing, 10 people from our recruited subjects were randomly picked for preliminary inter-rater, intra-rater, and inter-instrument reliabilities of these two ABGs, and these reliabilities were 0.80 or above. It should be noted that we struggled for which one is more securely stable and reliable for the IMU placement on the leg between the tibial tuberosity and the popliteal fossa below the knee joint line among these 10 subjects. It turned out that the tibial tuberosity is way more unreliable $(r<0.36)$ than the popliteal fossa below joint line $(r \geq 0.80)$ in terms of intra-subject and intra-rater reliabilities during static stance time.

After both ABG devices were secured and subjects being given initial verbal explanation and visual demonstration by the same investigator, the subjects were asked to stand on the non-dominant (left) foot on a level hard surface as comfortably as possible while lifting the dominant (right) foot off the floor to the level of medial malleolus of the left foot and maintaining an upright posture with hands by the side(Figure $1 \mathrm{~A}$ ). Then, the investigator for assessment synchronized these two ABGs (about 5 seconds) to start via a countdown to record while subjects maintained the SLS posture for 30 seconds. The right foot touching on the floor or on the left leg would be considered as failure of the test. If there was a failure, the test was re-administered after a 60 second rest period. Once completed the subjects were to wait five minutes to repeat the test. Data from two successful trials were processed and recorded through the attached $A B G$ software and transmitted wirelessly to a computer for data storage and later analysis.

Data Analysis: The IBM SPSS statistic software (Version 26, SPSS inc., Chicago, IL, USA) was used for this study. Collected data (range, angular velocity, and acceleration of sway on different directions) were presented with mean and stan- 
-dard deviation. These continuous dependent variables from two different locations (trunk and non-dominant lower extremity) were processed with the paired $t$ test. The level of statistical significance was set at 0.05 .
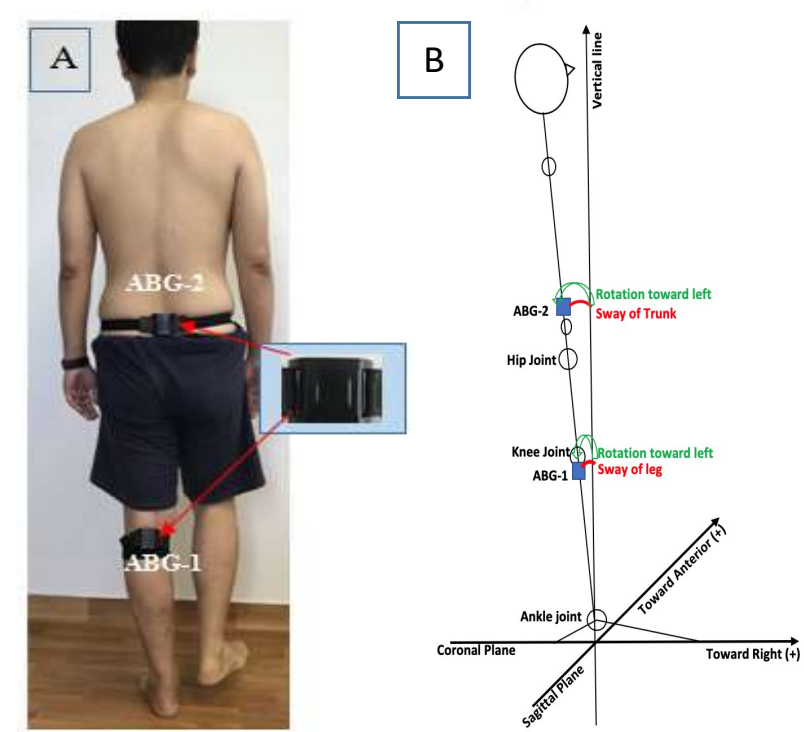

Fig. 1: A. The posterior view of a subject in single leg standing on the left side. The half-palm sized Alda Balance Gear $(A B G)$ is shown in the light blue box: $1^{\text {st }}$ one (ABG-1) placed at the popliteal fossa immediately below the knee joint line of the left leg, and $2^{\text {nd }}$ one (ABG-2) placed along the posterior midline at the L4-5 vertebral level. B. A human figure stands on the left foot on a 2-D floor - one on the sagittal plane (axis) from posterior () to anterior $(+)$, and other one on the frontal plane (axis) from the left (-) to the right (+). The vertical line through the center of base of support is near the ankle joint. ABG-1 and ABG-2 are able to catch leg and trunk movements respectively in 3-D space. On the 2-D (sagittal and coronal) planes, the figure stays toward the left and sways more posteriorly (red arcs); while on the axial (transverse) plane, both the leg and trunk rotate toward the left (green arcs).

\begin{tabular}{|c|c|c|c|c|}
\hline Parameters & Planes & Body Parts & Mean \pm SD & $P$ values \\
\hline \multirow[t]{6}{*}{ Range (degrees) } & ${ }^{*}$ Sagittal Plane & Leg & $-.19 \pm 1.18$ & .003 \\
\hline & & Trunk & $-1.59 \pm 1.41$ & \\
\hline & ${ }^{* *}$ Frontal Plane & Leg & $-.28 \pm .48$ & 159 \\
\hline & & Trunk & $-.06 \pm .29$ & \\
\hline & ***Axial Plane & Leg & $-25.77 \pm 75.33$ & 937 \\
\hline & & Trunk & $-27.11 \pm 81.20$ & \\
\hline \multirow[t]{6}{*}{ Angular Velocity } & Sagittal Plane & Leg & $.01 \pm .07$ & 0.037 \\
\hline & & Trunk & $.04 \pm .09$ & \\
\hline & Frontal Plane & Leg & $-.04 \pm .04$ & 689 \\
\hline & & Trunk & $-.03 \pm .05$ & \\
\hline & Axial Plane & Leg & $.07 \pm .17$ & 0.181 \\
\hline & & Trunk & $.01 \pm .13$ & \\
\hline \multirow[t]{6}{*}{ Acceleration (g-force) } & Sagittal Plane & Leg & $-.001 \pm .06$ & .022 \\
\hline & & Trunk & .05 \pm .09 & \\
\hline & Frontal Plane & Leg & $.29 \pm .12$ & 0.011 \\
\hline & & Trunk & $.36 \pm .08$ & \\
\hline & Axial Plane & Leg & $.97 \pm .03$ & 0.006 \\
\hline & & Trunk & $.94 \pm .02$ & \\
\hline
\end{tabular}

Int J Physiother Res 2020;8(5):3602-08. ISSN 2321-1822

\section{RESULTS}

As shown in Table 1, in the sagittal (anteriorposterior) plane, the trunk sway was significantly different than the standing leg in terms of range, velocity, and acceleration of sway (all $\mathrm{p}<.05$ ) - both leg and trunk swayed more posteriorly, but trunk did more range, faster speed, and greater acceleration than the leg. However, in both frontal (side-bending toward left or right) plane and axial (rotating toward left or right) plane, no statistically significant differences were found in range and velocity of sway between the leg and the trunk (all p>.05); but significant differences were still identified between the leg and the trunk in sway acceleration $(p<.05)$ - leg accelerated less than the trunk in the frontal plane, but more than the trunk in the axial plane. An interesting finding was that on the axial plane (Table 1 ), the ranges of rotation toward left for both leg $\left(25.77^{\circ}\right.$ in average) and trunk ( $27.11^{\circ}$ in average). There was no significant difference between the leg and trunk ( $p=.937)$ on the axial plane, but these values of range on this plane were much bigger than those on sagittal and frontal planes. By putting all planes together in a 3-D space, in general subjects swayed and rotated their bodies more leftward and backward when standing with the single leg on the left - an inverted pendulum with more range of sway in the sagittal plane (Table 1 and Figure 1B).
Table 1: Sway of Leg and Trunk in 3-D Spaces.

*on sagittal plane: + indicates swaying forward and - does backward

**on frontal plane: + indicates swaying rightward and - does leftward

***on axial plane: + indicates rotating rightward and - does leftward 


\section{DISCUSSION}

Ankle and hip joints for leg and trunk movements: During static stance, the ankle joint plays a critical role in providing fundamental and lowest joint stability by using its surrounding muscles to maintain the upright posture. However, when a static stance is influenced by a big perturbation and is no longer able to be controlled by the ankle joint, the hip joint could kick in to counteract any potential falling of the body [26]. It was found that postural corrective action occurs at multiple joints [27], primarily at hip and ankle joints $[5,8]$. At bipedal standing, leg and trunk coordinated to maintain the posture in angular excursion through the interactive feedback information from the ankle and hip joints [5]. The present study is in agreement with the early report [8] that in the upright posture with whole body weight on the dominant leg (single leg standing), the lower extremity provides more stability than the trunk. Regardless of unipedal or bipedal stance, Lower extremity muscles seemed to play more important role in maintaining the posture than trunk muscles [8].

Single leg stance on the non-dominant side: The SLS is commonly used to evaluate a person's balance ability, because the stability of single leg weight-bearing is often needed during daily activities, like making a turn when walking, or pick up an object from an overhead shelf [1]. Some researchers considered the SLS as a dynamic, rather than static, balance test [2]. Bigoni, Tarati and Gandolla et al (2017) [28] found that there was no difference in stance control during SLS between dominant and non-dominant legs in terms of postural frequency and regularity, but Promsri, Haid, and Enderolf et al (2018) [2] observed a significant difference in greater postural acceleration when standing on the non-dominant leg compared with on the dominant leg. In our present study, comparison of dominant and non-dominant legs were not compared, but by studying stance on the non-dominant leg, we did find that both the standing leg and trunk participated in the maintenance of upright posture, the sway parameters (range, angular velocity, and acceleration) are different between these two body segments.

Leg and trunk sway in 3-D space during single leg stance: As of how the leg and trunk response in different planes in a 3-D space during SLS has not been fully investigated before. Based on the current study, both the leg and trunks worked coordinately and individually (Figure 1B). Together, both segments moved to sway the body leftward a little bit and backward much more (Table 1) when the subject stood on the left leg. Individually, on the sagittal (anteriorposterior) plane, significant differences with the bigger range (-1.59 vs -0.19$)$, faster velocity, and greater acceleration were revealed on the leg segment than on the trunk segment. On the frontal(left-right) plane, both trunk and leg had tiny change in sway range (-0.06 vs -0.28$)$, but the trunk moved in quicker speed and greater acceleration than the leg. While on the axial plane, both trunk and leg had much bigger but not significant difference in range of sway $(-27.11$ vs -25.77$)$, but the trunk had more decreased acceleration compared with the leg. These together give an ellipsoid (instead of a circular) "inverted pendulum" movement-body sways more in sagittal plane after the body finds and secures a "stable" position by leaning and rotating the body leftward on both frontal and axial planes to certain degrees during static stance. This may indicate that young healthy subjects in this study may secure relative stability in two (frontal and axial) planes to ensure safe body sway in another (sagittal) plane. It for sure intrigues us to conduct the next similar study with a group of healthy older adults. In addition, we assume that movement of seeking postural stability on frontal and axial planes during the SLS could be resulted from the body's compensatory response to the lifted right lower extremity. Kinematically, rotation and sway toward left and posteriorly could lead the left hip joint and facet joints on the left side of the vertebrae in close-packed positions, which could make the body more rigid to fight against any potential balance disturbance [29]. All of these kinematic reactions might be coordinated and controlled by the central nervous system based on peripheral feedback of motion [4].

Study limitations: This study had a few limitations. First, in this study, we only explored the 
lower extremity and the trunk when the whole body was looked as a single unit in an upright posture. However, among the kinematic chain of joints from foot to the head, there are more than two body segments that involve balance maintenance. For more accurate data, more sensors for more body segments could be applied. Second, this study recruited only young healthy subjects. The results could not be generalized to the elderly population, but could be useful reference to the population. The third limitation is the that the recording started only 10 seconds after the SLS on the floor. We are not sure if 10 seconds were enough for a subject to adjust the body for "quiet" SLS. Lastly, it could be more informative if we did SLS comparison between the dominant leg and the non-dominant leg. In our future research plan, we will place sensors on both lower extremities and body parts with more body adjustment time (>10 seconds) in young persons, and also plan to conduct similar studies in older adults.

\section{CONCLUSION}

To our knowledge, this is a very first study that examined how two body segments response during static single leg stance time. The results of this study showed that during SLS on the non-dominant leg, the whole body sways more toward the stance side and posteriorly. Compared with the leg sway, the trunk displaying a larger range in faster speed and greater acceleration than the leg primarily in the sagittal (anterior-posterior) direction, and also quicker speed in axial plane, and greater acceleration in both axial and frontal planes. Such data may provide baseline information on how body segments may respond during SLS balance.

\section{Conflicts of interest: None}

\section{REFERENCES}

[1]. Springer BA, Marin R, Cyhan T, et al. Normative values for the unipedal stance test with eyes open and closed. J Geriatr Phys Ther. 2007; 30 (1):8-15. https://doi.org/10.1519/00139143-20070400000003 PMid:19839175

[2]. Promsri A, Haid T, Federolf PA. How does lower limb dominance influence postural control movements during single leg stance? Hum Movement Sci.2018; 58:165-174.

https://doi.org/10.1016/j.humov.2018.02.003 PMid:29448161
[3]. Lord SR, Sherrington C, Menz HB, Close JCT. Falls in Older People: Risk Factors and Strategies for Prevention, 2nd Edition. Pages 1-395. Cambridge University Press; 2007.

https://doi.org/10.1017/CBO9780511722233

[4]. Chiovetto E, Patane L, Pozzo T. Variant and invariant features characterizing natural and reverse wholebody pointing movements. Exp Brain Res. 2012; 218(3):419-431.

https://doi.org/10.1007/s00221-012-3030-y PMid:22370741

[5]. Hettich G, Asslander L, Gollhofer A, Mergner T. Human hip-ankle coordination emerging from multisensory feedback control. Hum Movement Sci. 2014; 37:123-146.

https://doi.org/10.1016/j.humov.2014.07.004 PMid:25150802

[6]. Jafari H, Pauelsen M, Roijezon U, Nyberg L, Nikolakopoulos G, Gustafsson T. On Internal Modeling of the upright postural control in elderly. 2018 IEEE International Conference on Robotics and Biomimetics, ROBIO 20182 July 2018, Article number 8665209, Pages 231-236.

https://doi.org/10.1109/ROBIO.2018.8665209

[7]. Wu G. Age-related differences in body segmental movement during perturbation stance in humans. Clin Biomech. 1998; 13(4-5): 300-307. https://doi.org/10.1016/S0268-0033(98)00068-0

[8]. Yu JH, Hong JH, Kim JS, Nam Y, Ryu JW, Lee DY, et al. Change of muscle activities in trunk, hip and leg muscles according to variable standing posture. Int J Appl Eng Res. 2014; 9(21):8433-8440.

[9]. Bohannon RW. A descriptive met-analysis of data from individuals at least 60 years of age. Top Geriatr Rehabil. 2006; 22(1):70-77. https://doi.org/10.1097/ 00013614-200601000-00010

[10]. Anderson SO, Atkeson CG, Hodgins JK. Coordinating feet in bipedal balance. Proceedings of the 2006 6th IEEE-RAS International Conference on Humanoid Robots, HUMANOIDS2006, Article number 4115669;624-628.

https://doi.org/10.1109/ICHR.2006.321339

[11]. Promsri A, Haid T, Federolf PA. Complexity, composition, and control of bipedal balance movement as the postural control system adapts to unstable support surface or altered feet positions. Neurosci. 2020; 430:113-124. https://doi.org/10.1016/ j.neuroscience.2020.01.031 PMid:32027995

[12]. Bonora G, Mancini M, Carpinella L, Chiari L, Ferrarin $M$, Nutt JG, et al. Investigation of Anticipatory Postural Adjustments during One-Leg Stance Using Inertial Sensors: Evidence from Subjects with Parkinsonism. Front Neurol. 2017. July 25. https://doi.org/10.3389/fneur.2017.00361 PMid:28790972 PMCid:PMC5524831

[13]. Oliveira MR, Vieira ER, Gil AWO, Fernandes KBP, Teixeira DC, Amorim CF, et al. One-legged stance sway of older adults with and without falls. PLOS ONE. 2018; 13(9): e0203887.

https://doi.org/10.1371/journal.pone.0203887 PMid:30222769 PMCid:PMC6141084 
Howe Liu, Yasser Salem, et all., COMPARISON OF TRUNK AND LEG SWAY DURING SINGLE LEG STANCE.

[14]. Karlsson A, Frykberg G. Correlation between force plate measures for assessment of balance. Clin Biotech. 2000; 15(5):365-369. https://doi.org/10.1016/S0268-0033(99)00096-0

[15]. Lee $\mathrm{CH}$, Sun TL. Evaluation of postural stability based on a force plate and inertial sensor during static balance measurements. J Physiol Anthropol. 2018; $37: 27$.

https://doi.org/10.1186/s40101-018-0187-5 PMid:30545421 PMCid:PMC6293511

[16]. Seimetz C, Tan D, Katayama R, Lockhart T. A comparison between methods of measuring postural stability: Force plates versus accelerometers. Biomed Sci Instrum. 2012; 48:386-392.

[17].Ma CZH, Wong DWC, Lam WK, Wan A, Lee W. Balance improvement effects of biofeedback systems with state-of-the-art wearable sensors: a systematic review. Sensors. 2016; 14:434.

https://doi.org/10.3390/s16040434

PMid:27023558 PMCid:PMC4850948

[18]. Gazit E, Buchman AS, Dawe R, Curran TA, Mirelman A, Giladi $N$, et al. What happens before the first step? A new approach to quantifying gait initiation using a wearable sensor. Gait Posture. 2020; 76:128135.

https://doi.org/10.1016/j.gaitpost.2019.11.007 PMid:31760316

[19]. Gordt K, Gerhardy T, Najafi B, Schwenk M. Effects of wearable sensor-based balance and gait training on balance, gait, and functional performance in healthy and patient populations: a systematic review and meta-analysis of randomized controlled trials. Gerontol. 2018;64:74-89.

https://doi.org/10.1159/000481454

PMid:29130977

[20].Ghislieri M, Gastaldi L, Pastorelli S, Tadano S, Agostini V. Wearable inertial sensors to assess standing balance: a systematic review. Sensor. 2019;19:4075.

https://doi.org/10.3390/s19194075

PMid:31547181 PMCid:PMC6806601

[21]. Noamani A, Nazarahari M, Lewicke J, Vette $A H$, Rouhani $\mathrm{H}$. Validity of using wearable inertial sensors for assessing the dynamics of standing balance. Med Eng Phys. 2020; 77:53-59.

https://doi.org/10.1016/j.medengphy.2019.10.018 PMid:31926830
[22]. Tanaka T, Hashimoto $N$, Nakata $M$, Ito $T$, Ino $S$, Ifukube $T$. Analysis of toe pressures under the foot while dynamic standing on one foot in healthy subjects: J Orthop Sports Phys Ther. 1996; 3:188-193. https://doi.org/10.2519/jospt.1996.23.3.188 PMid:8919397

[23]. Rethanya P, Babu KY, Mohanraj KG. 2018. Assessment of flat foot by plantar arch index using footprint in aged population. Drug Invent Today. 2018. 10(11):2142-2145.

[24]. Liu T, Inoue Y, Shibata K. A wearable sensor system for human motion analysis and human robot control. 2006 IEEE International Conference on Robotics and Biomimetics, ROBIO 20062006, Article number 4141837, 2006; Pages 43-48. https://doi.org/10.1109/ROBIO.2006.340276

[25]. Poitras I, Dupuis F, Bielmann M, Campeau-Lecours A, Mercier C, Bouyer LJ, et al. Validity and reliability of wearable sensors for joint angle estimation: $A$ systematic review. Sensors. 2019; 19:1555. https://doi.org/10.3390/s19071555 PMid:30935116 PMCid:PMC6479822

[26]. Kanamiya Y, Ota S, Sato D. Ankle and hip balance control on robotics and automations. Proceeding IEEE International Conference on Robotics and Automation. 2010. DOI: 10.1109/ ROBOT.2010.5509785.

https://doi.org/10.1109/ROBOT.2010.5509785

[27]. Dutt-Mazumder A, Challis J, Newell K. Maintenance of postural stability as a function of tilted base of support: Human movement science. 2016; 48:91101. https://doi.org/10.1016/j.humov.2016.04.010 PMid:27155961

[28].Bigoni M, Tarati M, Gandolla M, Augusti CA, Pedrocchi $A$, Torre $A L$, et al. Balance in young male soccer players: dominant versus non-dominant leg. Sport Sci Health. 2017; 13:253-258. https://doi.org/10.1007/s11332-016-0319-4

[29].Newmann DA. Kinesiology of the Musculoskeletal System: Foundations for Rehabilitations. 2nd edition. Mosby, 2009.

[30] Günther M, Wagner H. Dynamics of quiet human stance: computer simulations of a triple inverted pendulum model. Computer methods in biomechanics and biomedical engineering 8:819-34. https://doi.org/10.1080/10255842.2015.1067306 PMid:26214594

\section{How to cite this article:}

Howe Liu, Yasser Salem, Jingjie Zhou, Clayton Holmes, Myla Quiben. COMPARISON OF TRUNK AND LEG SWAY DURING SINGLE LEG STANCE. Int J Physiother Res 2020;8(5):3602-3608. DOI: 10.16965/ijpr.2020.151 